ADP-97-50/T277

\title{
In-medium kaon and antikaon properties in the quark-meson coupling model
}

\author{
K. Tsushima ${ }^{1}{ }^{*}$, K. Saito ${ }^{2} \nmid$ A. W. Thomas ${ }^{1}{ }^{\ddagger}$ and S. V. Wright ${ }^{1}{ }^{\S}$ \\ ${ }^{1}$ Department of Physics and Mathematical Physics \\ and Special Research Center for the Subatomic Structure of Matter, \\ University of Adelaide, SA 5005, Australia \\ ${ }^{2}$ Physics Division, Tohoku College of Pharmacy \\ Sendai 981, Japan
}

October 8, 2018

\begin{abstract}
The properties of the kaon, $K$, and antikaon, $\bar{K}$, in nuclear medium are studied in the quark-meson coupling (QMC) model. Employing a constituent quark-antiquark (MIT bag model) picture, their excitation energies in a nuclear medium at zero momentum are calculated within mean field approximation. The scalar, and the vector mesons are assumed to couple directly to the nonstrange quarks and antiquarks in the $K$ and $\bar{K}$ mesons. It is demonstrated that the $\rho$ meson induces different mean field potentials for each member of the isodoublets, $K$ and $\bar{K}$, when they are embedded in asymmetric nuclear matter. Furthermore, it is also shown that this $\rho$ meson potential is repulsive for the $K^{-}$meson in matter with a neutron excess, and renders $K^{-}$condensation less likely to occur.
\end{abstract}

PACS: 12.39.B, 14.40, 71.25J, 21.65, 13.75J

Keywords: In-medium kaon and antikaon properties, The quark-meson coupling model, Effective mass, kaon condensation, neutron star

*ktsushim@physics.adelaide.edu.au

${ }^{\dagger}$ ksaito@nucl.phys.tohoku.ac.jp

†athomas@physics.adelaide.edu.au

$\S_{\text {swright@physics.adelaide.edu.au }}$ 
The study of the properties of the kaon, $K$, and antikaon, $\bar{K}$, in a dense nuclear medium is one of the most exciting new directions in nuclear physics. Stimulated by the pioneering work of Kaplan and Nelson [1], intensive work has been performed about the possibility of the $K^{-}$meson condensation in a dense nuclear medium, and its effect on the properties of neutron stars [2] - [10]. In addition, many investigations have been made [11, 12 concerning $K N$ and $\bar{K} N$ interactions [13] - [19], and strangeness production in heavy ion collisions [12, 20, 21, 22, 23, 24, with a particular emphasis on the medium modification of the kaon and antikaon properties.

Although $K$ and $\bar{K}$ mesons are Goldstone bosons in the chiral limit, they are also expected to reveal a quark-antiquark $(q \bar{q})$ structure to some extent, because their mass is relatively heavy compared to that of the pion. Indeed, the naive constituent quark model has proven quite successful for studying the properties of $K$ and $\bar{K}$ mesons in free space [25].

However, not so many investigations have been performed on the properties of $K$ and $\bar{K}$ mesons in nuclear medium with explicit quark degrees of freedom [17. One of the main reasons for this is that there has been no appropriate model until recently, which can simultaneously describe the properties of the nuclear medium (finite nuclei), as well as hadron properties, based on quark degrees of freedom. It now seems possible to do this because of the recent development of the quark-meson coupling (QMC) model, which was initiated by Guichon [26]. This model has been successfully applied to investigate the properties of infinite nuclear matter [26] - 31] and finite nuclei [33] - [36], with some extension to incorporate the self-consistent variation of the meson masses [35]. Although the QMC model may be regarded as a general extension of Quantum Hadrodynamics (QHD) [37, the difference between the two models probably becomes distinctively clear when one investigates the properties of mesons in nuclear medium, where their quark structure plays a vital role.

Let the mean values of the meson fields, the scalar, the time component of the vector isoscalar, and the time component of the vector isovector in the third direction in isospin, be $\sigma, \omega$ and $b$, respectively, in a uniformly distributed nuclear medium. Here, we assume that the $K$ and $\bar{K}$ mesons are described by the static spherical MIT bag, as are the nucleons. We also suppose that the $\sigma, \omega$ and $\rho$ mesons only interact directly with the nonstrange quarks and antiquarks in the $K$ and $\bar{K}$ mesons. The Dirac equations for the corresponding wave functions for up and down quarks are given by:

$$
\begin{aligned}
& {\left[i \gamma \cdot \partial-\left(m_{q}-V_{\sigma}\right) \mp \gamma^{0}\left(V_{\omega}+\frac{1}{2} V_{\rho}\right)\right]\left(\begin{array}{c}
u \\
\bar{u}
\end{array}\right)=0,} \\
& {\left[i \gamma \cdot \partial-\left(m_{q}-V_{\sigma}\right) \mp \gamma^{0}\left(V_{\omega}-\frac{1}{2} V_{\rho}\right)\right]\left(\begin{array}{c}
d \\
\bar{d}
\end{array}\right)=0,}
\end{aligned}
$$

where $V_{\sigma}=g_{\sigma}^{q} \sigma, V_{\omega}=g_{\omega}^{q} \omega$ and $V_{\rho}=g_{\rho}^{q} b$ with $g_{\sigma}^{q}, g_{\omega}^{q}$ and $g_{\rho}^{q}$ being, respectively, the corresponding quark and meson coupling constants. Here we assume $\mathrm{SU}(2)$ symmetry. Thus, the current masses for the quarks and antiquarks follow the relation, $m_{q} \equiv m_{u}=m_{d}=m_{\bar{u}}=m_{\bar{d}}$. The normalized, static solution for the ground state for a nonstrange quark or antiquark in the kaon or antikaon may be written as:

$$
\psi_{i}(\vec{r})=N_{i} e^{-i \epsilon_{i} t / R_{K}^{\star}} \phi_{i}(\vec{r}), \quad \text { for } i=u, \bar{u}, d, \bar{d}
$$

where $N_{i}$ and $\phi_{i}(\vec{r})$ are respectively the normalization factor and the corresponding spatial part of the wave function [39]. The bag radius in medium, $R_{K}^{\star}$, which depends on the hadron species in which the quarks belong, will be determined self-consistently through Eqs. (6) and (7) 
similarly to those for the octet baryons [33, 35, 36]. The quark eigenenergies in units of $1 / R_{K}^{\star}$, $\epsilon_{i}(i=u, \bar{u}, d, \bar{d})$ in Eq. (3), are given by

$$
\left(\begin{array}{c}
\epsilon_{u} \\
\epsilon_{\bar{u}}
\end{array}\right)=\Omega^{\star} \pm R_{K}^{\star}\left(V_{\omega}+\frac{1}{2} V_{\rho}\right) \quad \text { and } \quad\left(\begin{array}{c}
\epsilon_{d} \\
\epsilon_{\bar{d}}
\end{array}\right)=\Omega^{\star} \pm R_{K}^{\star}\left(V_{\omega}-\frac{1}{2} V_{\rho}\right),
$$

where $\Omega^{\star}=\sqrt{x^{\star 2}+\left(R_{K}^{\star} m_{q}^{\star}\right)^{2}}$, with $m_{q}^{\star}=m_{q}-g_{\sigma}^{q} \sigma$. The quark eigenfrequency in medium, $x^{\star}$, is determined by the usual, linear boundary condition [32, 39]. Then the excitation energies for the $K$ and $\bar{K}$ mesons with zero momenta, $\omega_{i}\left(i=K^{+}, K^{-}, K^{0}, \bar{K}^{0}\right)$, are given by

$$
\left(\begin{array}{c}
\omega_{K^{+}} \\
\omega_{K^{-}}
\end{array}\right)=m_{K}^{\star} \pm\left(V_{\omega}+\frac{1}{2} V_{\rho}\right) \quad \text { and } \quad\left(\begin{array}{c}
\omega_{K^{0}} \\
\omega_{\bar{K}^{0}}
\end{array}\right)=m_{K}^{\star} \pm\left(V_{\omega}-\frac{1}{2} V_{\rho}\right)
$$

where, the effective mass of the $K$ and $\bar{K}$ mesons, $m_{K}^{\star}=m_{\bar{K}}^{\star}$, is calculated using the MIT bag model:

$$
\begin{aligned}
& m_{K}^{\star}=\frac{\Omega^{\star}+\Omega_{s}-z_{K}}{R_{K}^{\star}}+\frac{4}{3} \pi R_{K}^{\star 3} B, \\
& \left.\frac{\partial m_{K}^{\star}}{\partial R}\right|_{R=R_{K}^{\star}}=0
\end{aligned}
$$

with the strange-quark energy in units of $1 / R_{K}^{\star}, \Omega_{s}=\sqrt{x_{s}^{2}+\left(R_{K}^{\star} m_{s}\right)^{2}}$, and $z_{K}$ in Eq. (6) parametrizes the sum of the center-of-mass and gluon fluctuation effects.

After self-consistent calculation, the effective mass of the $K$ and $\bar{K}$ mesons, $m_{K}^{\star}$, can be parametrized in the applied scalar field [32, 33, 35, 36]:

$$
m_{K}^{\star} \equiv m_{K}-g_{\sigma}^{K}(\sigma) \sigma \simeq m_{K}-\frac{1}{3} g_{\sigma}^{N}\left[1-\frac{a_{K}}{2}\left(g_{\sigma}^{N} \sigma\right)\right] \sigma,
$$

where $g_{\sigma}^{N}$ is the nucleon and $\sigma$ meson coupling constant in free space $(\sigma=0)$ [32, 36, and the relation, $g_{\sigma}^{K}=\frac{1}{3} g_{\sigma}^{N}$, involves an error less than $0.5 \%$ [33, 35, 36]. (The quantity, $a_{K}$, in Eq. (8) is found to be $a_{K}=6.6 \times 10^{-4} \mathrm{MeV}^{-1}$.) In this study, we chose the values, $m_{u}=m_{d}=5$ $\mathrm{MeV}$ and $m_{s}=250 \mathrm{MeV}$, for the current quark masses, and $R_{N}=0.8 \mathrm{fm}$ for the bag radius of the nucleon in free space. Other inputs and parameters used, and some of the quantities calculated in the present study, are listed in Table 11. (Note that $r_{q}$ is the root-mean-square (r.m.s.) charge radius calculated using the MIT bag model wave functions obtained by solving the set of equations including the strange quark.) We stress that while the model has a number of parameters, only three of them, $g_{\sigma}^{q}, g_{\omega}^{q}$ and $g_{\rho}^{q}$, are adjusted to fit nuclear data - namely the saturation energy and density of symmetric nuclear matter and the bulk symmetry energy. None of the results for nuclear properties depend strongly on the choice of the other parameters - for example, the relatively weak dependence of the final results on the chosen values of the current quark mass and bag radius is shown explicitly in Refs. 32, 33.

Boosting the $K$ and $\bar{K}$ bags in a uniformly distributed vector field, we can find the dispersion relation for the $K$ and $\bar{K}$ mesons moving with momentum $\vec{k}$ as in Ref. [20]:

$$
\left(\begin{array}{c}
\omega_{K^{+}}(\vec{k}) \\
\omega_{K^{-}}(\vec{k})
\end{array}\right)=\sqrt{m_{K}^{\star}+\vec{k}^{2}} \pm\left(V_{\omega}+\frac{1}{2} V_{\rho}\right) \quad \text { and } \quad\left(\begin{array}{c}
\omega_{K^{0}}(\vec{k}) \\
\omega_{\bar{K}^{0}}(\vec{k})
\end{array}\right)=\sqrt{m_{K}^{\star}+\vec{k}^{2}} \pm\left(V_{\omega}-\frac{1}{2} V_{\rho}\right)
$$


Table 1: Inputs, parameters and some of the quantities calculated in the present study. The quantities with star, ${ }^{\star}$, are those quantities calculated at normal nuclear density, $\rho_{0}=0.15 \mathrm{fm}^{-3}$. The values for the bag constant, and current quark masses are respectively, $B=(170.0 \mathrm{MeV})^{4}$, and $m_{u}=m_{d}=5 \mathrm{MeV}$ and $m_{s}=250 \mathrm{MeV}$.

\begin{tabular}{|l|lllllll|}
\hline & $m(\mathrm{MeV})$ & $z_{N, K}$ & $R(\mathrm{fm})$ & $r_{q}(\mathrm{fm})$ & $m^{\star}(\mathrm{MeV})$ & $R^{\star}(\mathrm{fm})$ & $r_{q}^{\star}(\mathrm{fm})$ \\
\hline $\mathrm{N}$ & 939.0 (input) & 3.295 & 0.800 (input) & 0.582 & 754.6 & 0.786 & 0.594 \\
$\mathrm{~K}$ & 493.7 (input) & 3.295 & 0.574 & 0.412 & 430.5 & 0.572 & 0.418 \\
\hline
\end{tabular}

This is equivalent to the dispersion relation which is given by the gauge invariant effective Lagrangian density at the hadronic level [9, 10]:

$$
\mathcal{L}=\left[\left(\partial_{\mu}+i g_{\omega}^{K} \omega_{\mu}+i g_{\rho}^{K} \frac{\tau_{3}}{2} \rho_{\mu}\right) K\right]^{\dagger}\left[\left(\partial^{\mu}+i g_{\omega}^{K} \omega^{\mu}+i g_{\rho}^{K} \frac{\tau_{3}}{2} \rho^{\mu}\right) K\right]-m_{K}^{\star 2} \bar{K} K+\mathcal{L}_{\text {matter }}
$$

where $K=\left(\begin{array}{c}K^{+} \\ K^{0}\end{array}\right)$ is the second quantized kaon field with $\bar{K}=K^{\dagger}$, and $\mathcal{L}_{\text {matter }}$ is the Lagrangian density of a nuclear system [32, 33, 35]. In our approach, the effective mass, $m_{K}^{\star}$, is calculated using the MIT bag model, Eq. (6) and the result is very well approximated by Eq. (8).

In Fig. 1, we show the binding energy per nucleon, $(E / A)-m_{N}$, versus baryon density. Neutron matter and nuclear matter in Fig. 1 denote matter with proton fractions 0 and 0.5 (symmetric matter), respectively. This notation will be used hereafter. The coupling constants, $g_{\sigma}^{N}, g_{\omega}^{N}$ and $g_{\rho}^{N}$ are determined so as to reproduce the saturation properties of symmetric nuclear matter at normal nuclear density, $\rho_{0}$, namely, the binding energy per nucleon, $-15.7 \mathrm{MeV}$, and bulk symmetry energy, $35.0 \mathrm{MeV}$. The values for the coupling constants determined in this way are given in Table 2. Using these parameters, the nuclear incompressibility, $K$, is calculated to be $K=279.2 \mathrm{MeV}$, which is well within the empirically required range.

Table 2: Values of the coupling constants determined required to reproduce the saturation properties of symmetric nuclear matter at normal nuclear density, $\rho_{0}=0.15 \mathrm{fm}^{-3}$. For the relation between the coupling constants, $g_{\sigma}^{q}$ and $g_{\sigma}^{N}$, or, the origin of the constant factor between them, see Refs. 32, 33.

\begin{tabular}{|c|c|c|c|}
\hline$g_{\sigma}^{q}=g_{\sigma}^{N} /(3 \times 0.483)$ & $\left(g_{\sigma}^{N}\right)^{2} / 4 \pi$ & $\left(g_{\omega}^{N}\right)^{2} / 4 \pi=\left(3 g_{\omega}^{q}\right)^{2} / 4 \pi$ & $\left(g_{\rho}^{N}\right)^{2} / 4 \pi=\left(g_{\rho}^{q}\right)^{2} / 4 \pi$ \\
\hline 5.69 & 5.39 & 5.30 & 6.93 \\
\hline
\end{tabular}

In Fig. 2 we show the effective masses of the $K$ and $\bar{K}$ mesons and the nucleon, as well as the scalar and vector mean field potentials. For the present we have omitted any effects of hyperons in the background nuclear medium. We observe that neither the effective mass of the $K$ and $\bar{K}$ mesons, $m_{K}^{\star}$, nor that of the nucleon decreases linearly as the density increases. This feature may be ascribed to the quark structure in the present approach, and clearly differs from the linear decrease in QHD [37]. This behaviour can be also understood from the 
parametrization, Eq. (8). $g_{K \omega}^{q} \omega$ in Fig. 2 denotes the vector potential for the nonstrange quarks and antiquarks in the $K$ and $\bar{K}$ mesons, and we will explain this below, in connection with the $K^{+} N$ potential.

It is known empirically that the $K^{+} N$ potential is slightly repulsive if one wants to be consistent with the $K^{+} N$ scattering length, and the corresponding value at $\rho_{B}=0.16 \mathrm{fm}^{-3}$ is estimated to be about $20 \mathrm{MeV}$ [38. On the other hand, the present model gives a very slightly attractive $K^{+} N$ potential. We believe that this minor shortcoming has its origin in the deficiencies of the bag model in dealing with the Goldstone nature of the $K$ and $\bar{K}$ mesons. As a phenomenological means of compensation for this we rescale the coupling constant, $g_{\omega}^{q}$, to reproduce the $K^{+} N$ potential, $+20 \mathrm{MeV}$, at $\rho_{B}=0.16 \mathrm{fm}^{-3}$. That is, we use, $g_{K \omega}^{q}=1.4^{2} \times g_{\omega}^{q}$, for the $\omega$ meson coupling constant to the nonstrange quark (and antiquark) in the $K$ and $\bar{K}$ mesons. Note that the coupling constant, $g_{K \omega}^{q}$, is the only parameter adjusted in the present study. None of our qualitative conclusions would be altered if we did not make this adjustment. The $\omega$ mean field vector potential calculated using this rescaled coupling constant is denoted by $g_{K \omega}^{q} \omega$ in Fig. 2.

In Fig. 3, we show the calculated kaon excitation energies at zero momentum versus the baryon density. It is interesting to notice that although the excitation energies for the isodoublet members, $K^{+}$and $K^{0}$, are degenerate in symmetric nuclear matter, this is no longer true in asymmetric nuclear matter. This is a consequence of the $\rho$ meson which couples to the nonstrange quarks (antiquarks) in the kaon (antikaon).

Next, we show the antikaon excitation energies in Fig. 4 , as well as the difference of the calculated chemical potentials for the neutron and proton, $\mu_{n}-\mu_{p}$, which is calculated by

$$
\mu_{n}-\left.\mu_{p} \simeq \frac{E(Z, N)}{\partial N}\right|_{Z}-\left.\frac{E(Z, N)}{\partial Z}\right|_{N}=-\frac{\partial}{\partial x}\left(\frac{E(Z, N)}{A}\right),
$$

where $Z, N(A=Z+N)$ and $E(Z, N) / A$ are, respectively, the proton number, neutron number and the total energy per nucleon with proton fraction, $x \equiv Z / A$. Because we have not included any effects which are expected to lower the values of $\mu_{n}-\mu_{p}$, or raise the critical density for the onset of $K^{-}$meson condensation (such as hyperons or muons [5, 9, 40], the $\delta$ meson [10], non-zero momentum for the $K^{-}$mesons due to the thermal fluctuations or shortrange correlations), the critical density found for each case may be regarded as a lower limit. Again the excitation energies for the isodoublet members, $K^{-}$and $\bar{K}^{0}$, are no longer degenerate in asymmetric nuclear matter. In particular, the excitation energy for the $K^{-}$meson at a fixed density increases as the neutron fraction increases. Thus, the $\rho$ meson plays a role in making $K^{-}$meson condensation less favorable to occur in a matter with a larger neutron excess. This effect of the $\rho$ field on the $K^{-}$meson, certainly should be taken into account when one studies $K^{-}$-condensation and its effect on the properties of neutron stars.

If we use the parametrization of Eq. (8), together with the explicit expressions for the vector mean fields using the isoscalar $\left(\rho_{B}=\rho_{p}+\rho_{n}\right)$ and isovector $\left(\rho_{3} \equiv \rho_{p}-\rho_{n}\right)$ baryon densities, the excitation energy for the $K^{-}$meson at zero momentum, $\omega_{K^{-}}$, can be expressed as:

$$
\begin{aligned}
\omega_{K^{-}} & \equiv m_{K}^{\star}-V_{\omega}^{K}-\frac{1}{2} V_{\rho}, \\
& \simeq m_{K}-g_{\sigma}^{K}\left[1-\frac{a_{K}}{2}\left(g_{\sigma}^{N} \sigma\right)\right] \sigma-\frac{g_{\omega}^{K} g_{\omega}^{N}}{m_{\omega}^{2}} \rho_{B}-\frac{g_{\rho}^{K} g_{\rho}^{N}}{4 m_{\rho}^{2}} \rho_{3},
\end{aligned}
$$


where $g_{\sigma}^{K}=\frac{1}{3} g_{\sigma}^{N}, g_{\omega}^{K}=1.4^{2} \times g_{\omega}^{q}=1.4^{2} \times \frac{1}{3} g_{\omega}^{N}$ and $g_{\rho}^{K}=g_{\rho}^{q}=g_{\rho}^{N}$. For a rough estimate of the $K^{-}$excitation energy up to $\rho_{B} \sim \rho_{0}$, one can use the approximate value for the scalar field $g_{\sigma}^{N} \sigma \simeq 200 \rho_{B} / \rho_{0}(\mathrm{MeV})$.

In summary, we have studied the properties of kaon and antikaon in nuclear matter, using the QMC model for the first time. Although the model should eventually incorporate chiral symmetry in order to treat the kaon and antikaon as pseudo-Goldstone bosons, our present emphasis was on the role of the $\rho$ meson in an asymmetric nuclear medium. In particular, in matter with a neutron excess, or with a negative isovector density, the $\rho$ meson induces a repulsive potential for the $K^{-}$meson. This effect should certainly be taken into account in investigations of the properties of neutron stars, and kaon flow in heavy ion collisions, where it has so far been omitted [22]. Indeed, it may be possible to test our estimate of this effect by calculating the $\bar{K}^{0}$ and $K^{-}$flow in heavy ion collisions. In the present study, we have not included any effects which are expected to lower the chemical potential of the electron such as a non-zero hyperon density [5, 9, 40]. For a more realistic study, it will be necessary to include self-consistently the effect of the hyperons in calculating scalar and vector fields. In that case, it is possible that the quark structure of the hadrons, which appears mainly in a non-linear variation of their effective masses, may give nontrivial effects. In particular, our further interest is whether the QMC model can avoid the negative effective mass problem for the nucleon, that was discussed by Schaffner and Mishustin [9].

The authors would like to thank G.Q. Li, T. Tatsumi and A.G. Williams for helpful discussions.

This work is supported by the Australian Research Council and the Japan Society for the Promotion of Science.

\section{References}

[1] D.B. Kaplan and A.E. Nelson, Phys. Lett. B 175 (1986) 57; B 179 (1986) 409(E);

A.E. Nelson and D.B. Kaplan, Phys. Lett. B 192 (1987) 193.

[2] G.E. Brown, K. Kubodera and M. Rho, Phys. Lett. B 192 (1987) 273;

G.E. Brown, K. Kubodera, M. Rho and V. Thorsson, Phys. Lett. B 291 (1992) 355;

H. Yabu, S. Nakamura, F. Myhrer and K. Kubodera, Phys. Lett. B 315 (1993) 17.

[3] Toshitaka Tatsumi, Prog. Theor. Phys. 80 (1988) 22; Prog. Theor. Phys. Supp. 120 (1995) 111

H. Fujii, T. Maruyama, T. Muto and T. Tatsumi, Nucl. Phys. A 597 (1996) 645.

[4] J. Schaffner, A. Gal, I. Mishustin, H. Stöcker, W. Greiner, Phys. Lett. B 334 (1994) 268.

[5] R. Knorren, M. Prakash, P.J. Ellis, Phys. Rev. C 52 (1995) 3470;

P.J. Ellis, J.M. Lattimer, M. Prakash, Comments on Nucl. and Part. Phys. 22 (1996) 63.

[6] Chang-Hwan Lee, Phys. Rep. 275 (1996) 255, and references therein.

[7] G.E. Brown, Mannque Rho, Nucl. Phys. A 596 (1996) 503.

[8] Madappa Prakash, Ignazio Bombaci, Manju Prakash, Paul J. Ellis, James M. Lattimer, Roland Knorren, Phys. Rep. 280 1, and references therein. 
[9] J. Schaffner and I. Mishustin, Phys. Rev. C 53 (1996) 1416.

[10] Z.G. Dai, K.S. Cheng, Phys. Lett. B 401 (1997) 219.

[11] G.E. Brown, Mannque Rho, Phys. Rep. 269 (1996) 333, and references therein.

[12] G.Q. Li, C.-H. Lee and G.E. Brown, nucl-th/9706057, and refernces therein; nucl-th/9709024; Phys. Rev. Lett. 79 (1997) 5214.

[13] P.B. Siegel, W.B. Kaufmann, W.R. Gibbs, Phys. Rev. C 31 (1985) 2184.

[14] G.E. Brown, C.B. Dover, P.B. Siegel and W. Weise, Phys. Rev. Lett. 26 (1988) 2723.

[15] C.M. Chen, D.J. Ernst, Phys. Rev. C 45 (1992) 2019.

[16] J.C. Caillon, J. Labarsouque, Phys. Lett. B 295 (1992) 21; Phys. Rev. C 45 (1992) 2503; Nucl. Phys. A 572 (1994) 649; Nucl. Phys. A 892 (1995) 609.

[17] M. Lutz, A Steiner, W. Weise, Nucl. Phys. A 574 (1994) 755.

[18] T. Waas, N. Kaiser, W. Weise, Phys. Lett. B 379 (1996) 755;

T. Waas, M. Rho and W. Weise, Nucl. Phys. A 617 (1997) 449.

[19] M. Lutz, GSI-Preprint-97-55, nucl-th/9709073.

[20] G.E. Brown, C.M. Ko and K. Kubodera, Z. Phys. A 341 (1992) 301.

[21] C.M. Ko, X.S. Fang, and Y.M. Zheng, in Proc. of the International Workshop XXI on Gross Properties of Nuclei and Nuclear Excitations, Hirschegg, Kileinwalsertal, Austria, January 18 - 23, 1993, edited by H. Feldmeire, (1993) 29;

X.S. Fang, C.M. Ko, G.Q. Li and Y.M. Zheng, Phys. Rev. C 49 (1994) R608;

G.Q. Li, C.M. Ko, X.S. Fang, Phys. Lett. B 329 (1994) 149;

C.M. Ko and G.Q. Li, J. Phys. G 22 (1996) 1673, and references therein;

G.Q. Li, C.M. Ko and W.S. Chung, nucl-th/9710032.

[22] G.Q. Li, C.M. Ko, Nucl. Phys. A 594 (1995) 460; and private communication G.Q. Li.

[23] Volker Koch, Phys. Lett. B 351 (1995) 29.

[24] E.L. Bratkovskaya, W. Cassing, U. Mosel, Nucl. Phys. A 622 (1997) 593.

[25] T. DeGrand, R.L. Jaffe, K. Johnson, and J. Kiskis, Phys. Rev. D 12 (1975) 2060;

See, for example, F.E. Close, An Introduction to Quarks and Partons, Academic Press Inc. (London) Ltd, (1982).

[26] P.A.M. Guichon, Phys. Lett. B 200 (1988) 235.

[27] K. Saito and A.W. Thomas, Phys. Lett. B 327 (1994) 9; Phys. Rev. C 52 (1995) 2789.

[28] K. Saito and A.W. Thomas, Phys. Rev. C 51 (1995) 2757.

[29] X. Jin and B.K. Jennings, Phys. Lett. B 374 (1996) 13; Phys. Rev. C 54 (1996) 1427; Phys. Rev. C 55 (1997) 1657; nucl-th/9709074;

H. Müller and B.K. Jennings, nucl-th/9706049. 
[30] R.M. Aguirre, M. Schvellinger, Phys. Lett. B 400 (1997) 245; nucl-th/9704016; nuclth/9709031.

[31] P.K. Panda, A. Mishra, J.M. Eisenberg, W. Greiner, nucl-th/9705045.

[32] P.A.M. Guichon, K. Saito, E. Rodionov and A.W. Thomas, Nucl. Phys. A 601 (1996) 349; P.A.M. Guichon, K. Saito and A.W. Thomas, Australian Journal of Physics 50 (1997) 115.

[33] K. Saito, K. Tsushima and A.W. Thomas, Nucl. Phys. A 609 (1996) 339; nucl-th/9608062, in Proc. of the Int. National Symposium on Non-Nucleonic Degrees of Freedom Detected in Nucleus, Sep. 2-5, 1996, Osaka, Japan, World Scientific (1997) 93;

D.H. Lu, A.W. Thomas, K. Tsushima, A.G. Williams, K. Saito, nucl-th/9706043, to appear in Phys. Lett. B.

[34] P.G. Blunden and G.A. Miller, Phys. Rev. C 54 (1996) 359.

[35] K. Saito, K. Tsushima and A.W. Thomas, Phys. Rev. C 55 (1997) 2637;

Phys. Rev. C 56 (1997) 566; Phys. Lett. B 406 (1997) 287; nucl-th/9608060, in Proc. of the Int. National Symposium on Non-Nucleonic Degrees of Freedom Detected in Nucleus, Sep. 2-5, 1996, Osaka, Japan, World Scientific (1997) 345;

K. Saito, nucl-th/9705019, in Proc. of the International mini workshop on nuclear medium effect via nucleon induced reactions, March 21 - 22, 1997, Kyoto, Japan, Genshikaku kenkyu (Japan), vol. 42, (1997) 181;

A.W. Thomas, nucl-th/9707005, to be published in Proc. of the Int. Conf. on Quark Lepton Nuclear Physics, Osaka, May 20 - 23, 1997;

K. Saito, K. Tsushima and A.W. Thomas, ADP-97-33/T266, nucl-th/9709040, to appear in Mod. Phys. Lett. A.

[36] K. Tsushima, K. Saito and A.W. Thomas, Phys. Lett. B 411 (1997) 9; (E) technically caused by printing, to appear in Phys. Lett. B;

K. Tsushima, K. Saito, J. Haidenbauer, and A.W. Thomas, ADP-97-26/T261, nuclth/9707022, to appear in Nucl. Phys. A.

[37] J.D. Walecka, Ann. Phys. (N.Y.) 83 (1974) 491;

B.D. Serot and J.D. Walecka, Adv. Nucl. Phys. 16 (1986) 1.

[38] G.Q. Li, nucl-th/9710008, Sec. III B;

G.Q. Li, C.-H. Lee, and G. E. Brown, Phys. Rev. Lett. 79 (1997) 5214.

[39] For example, K. Tsushima, T. Yamaguchi, Y. Kohyama and K. Kubodera, Nucl. Phys. A489 (1988) 557, Appendix C.

[40] N.K. Glendenning, Phys. Lett. 114B (1982) 392; Astrophys. J. 293 (1985) 470; Z. Phys. A 326 (1987) 57. 


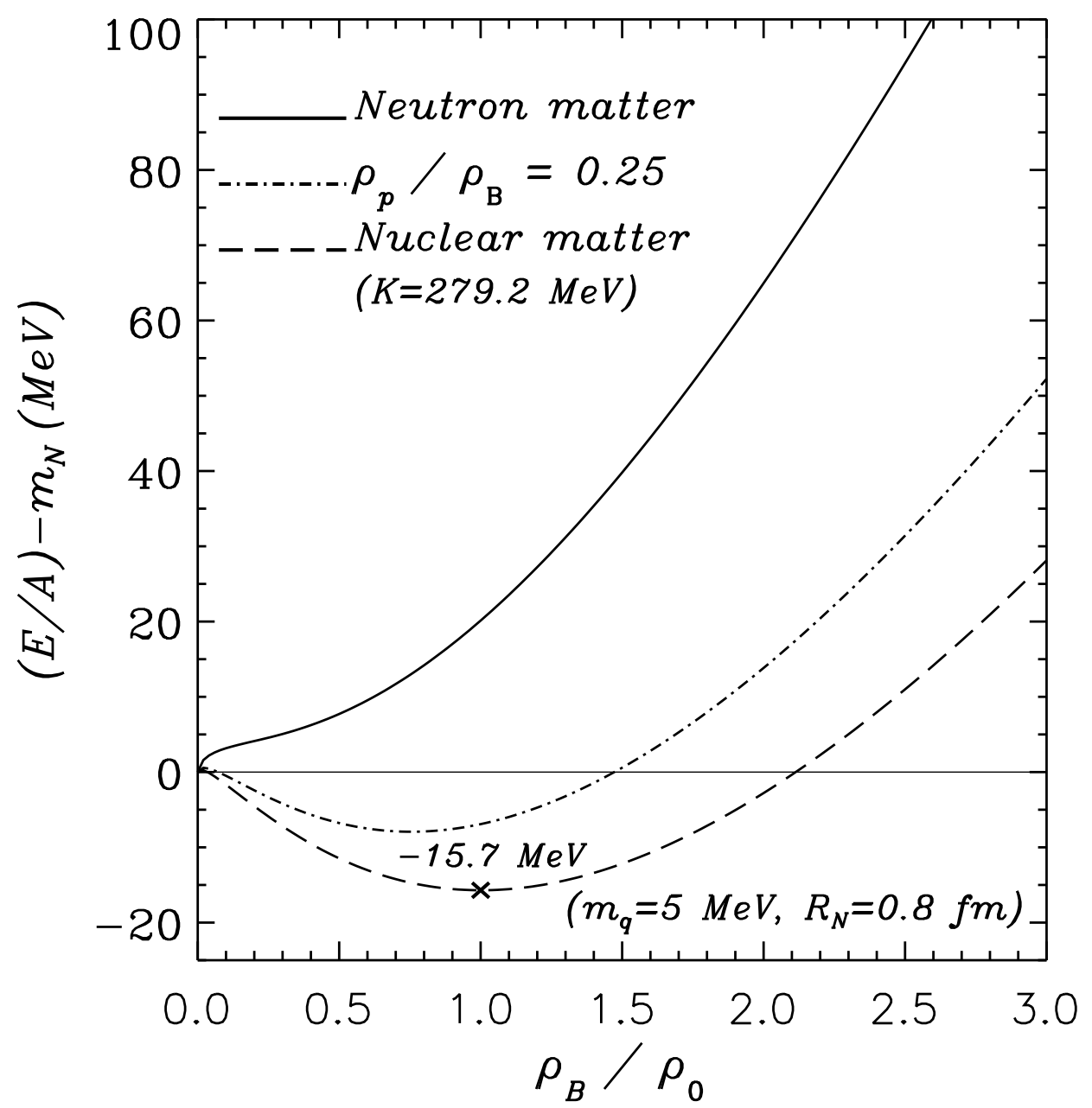

Figure 1: Binding energy per nucleon for matter with different proton fractions. Neutron matter and nuclear matter denote matter with proton fractions 0 and 0.5 , respectively. $K=279.2$ $\mathrm{MeV}$ is the value obtained for the nuclear incompressibility in the present model. 


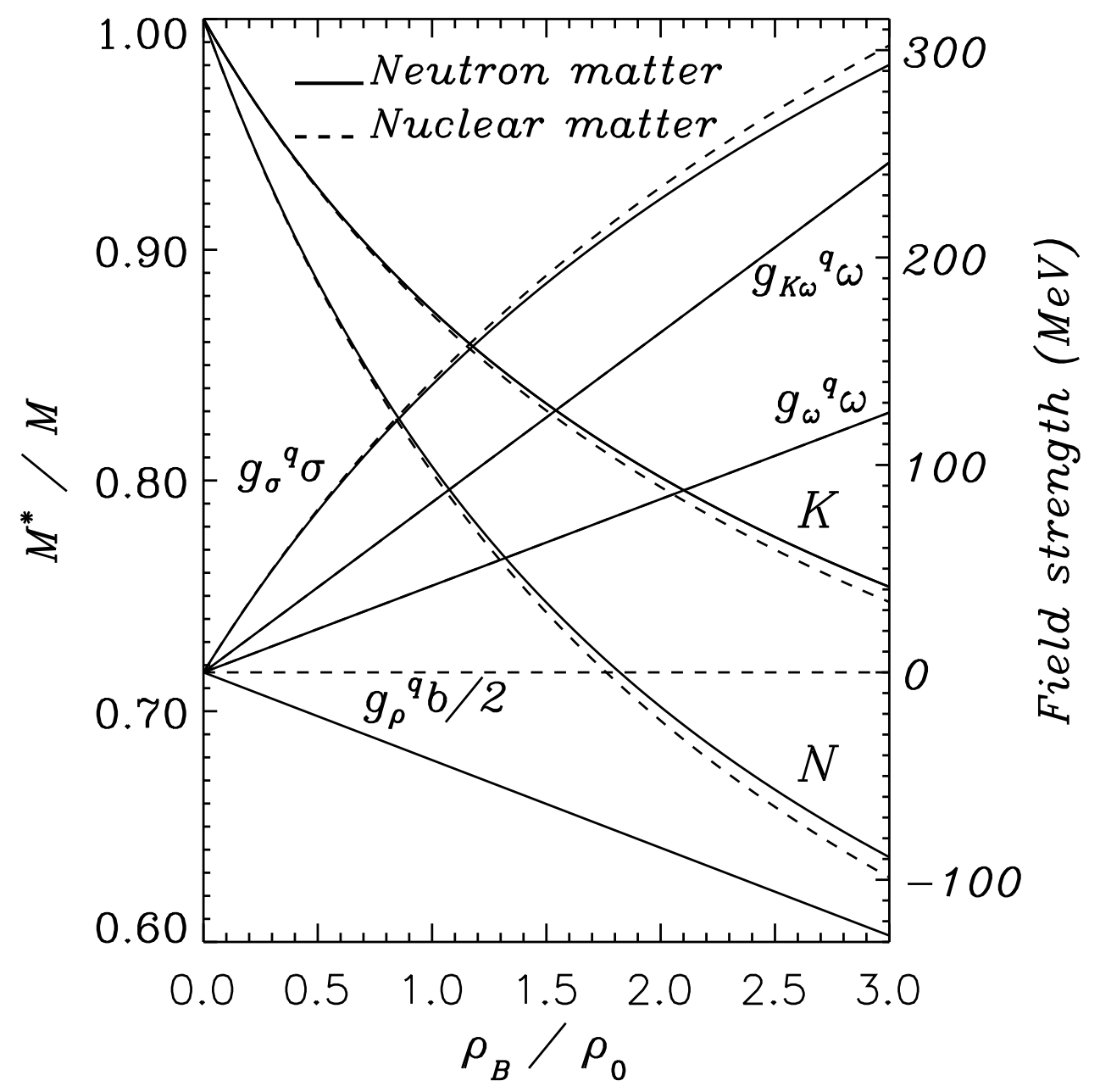

Figure 2: Effective masses of the nucleon, $K$ (and $\bar{K}$ ) meson and the mean field potentials for the nonstrange quarks and antiquarks. $g_{K \omega}^{q} \omega$ is the $\omega$ meson mean field potential for the nonstrange quarks and antiquarks in the $K$ and $\bar{K}$ mesons. 


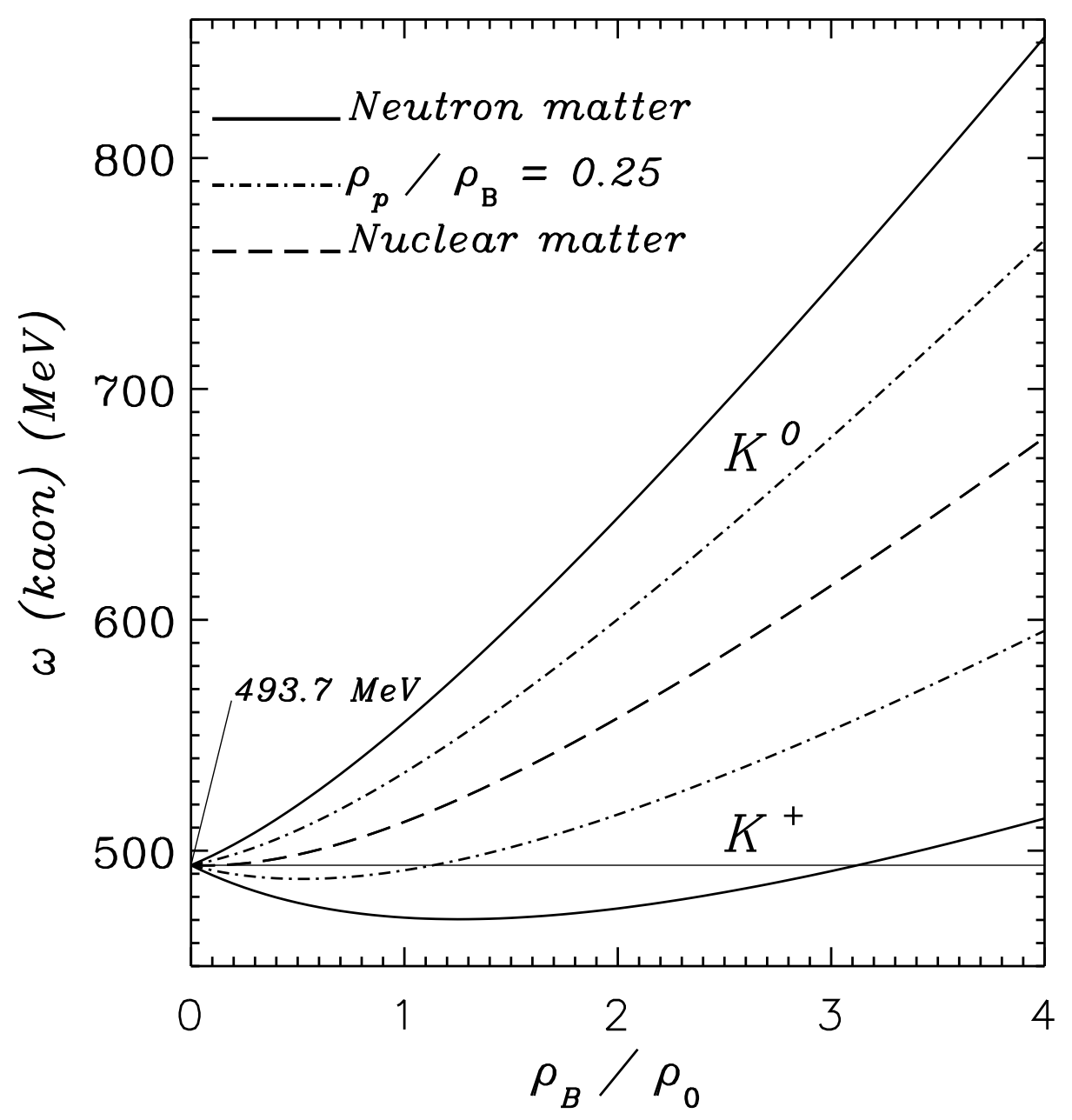

Figure 3: Kaon excitation energies at zero momentum. 


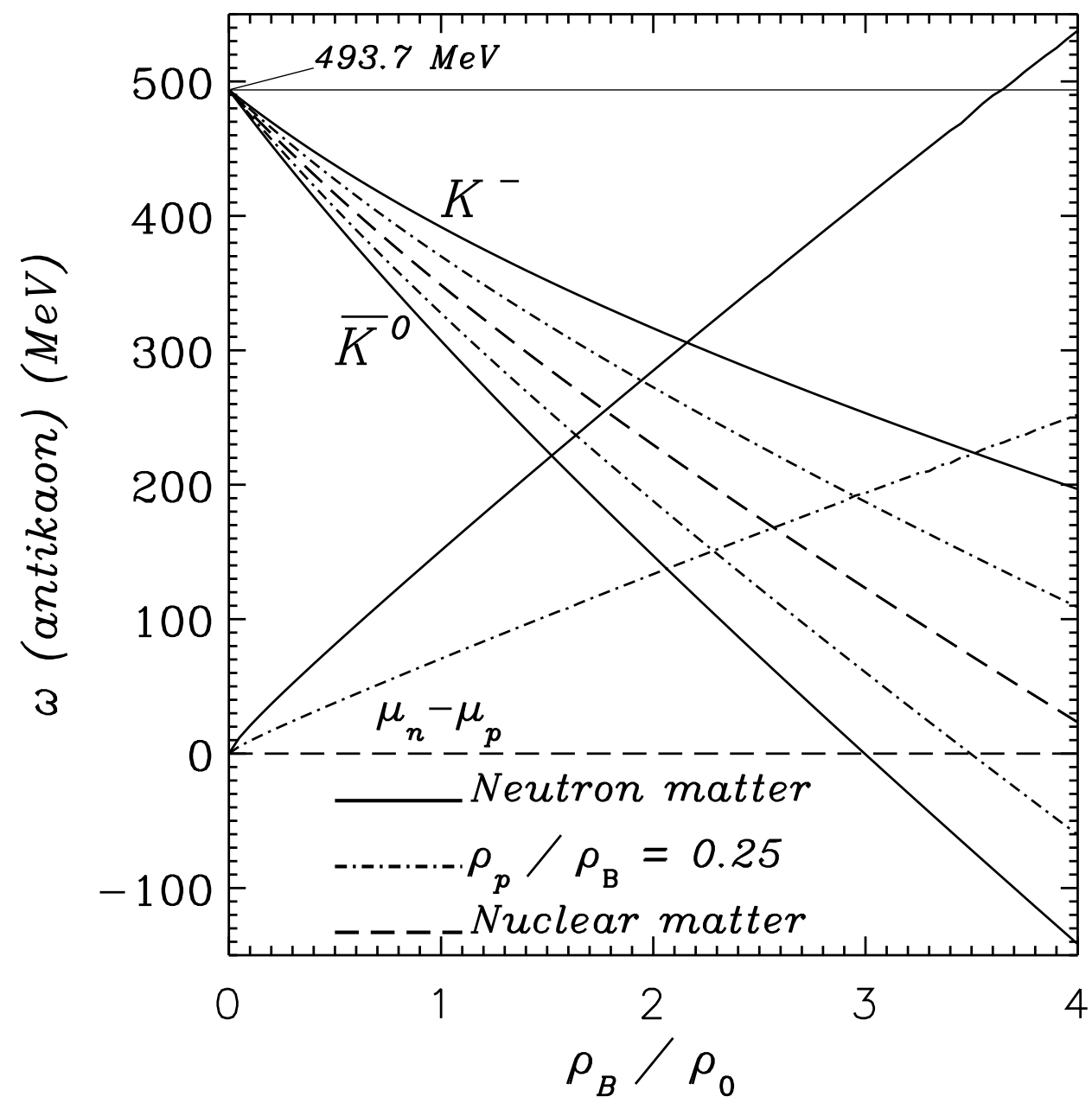

Figure 4: Antikaon excitation energies at zero momentum, and the difference of the calculated chemical potentials for the neutron and proton, $\mu_{n}-\mu_{p}$. 